URINARY INCONTINENCE

\section{Detrusor activity predicts renal damage}

The avoidance of renal failure is a primary objective of the long-term management of patients with a previous spinal cord injury (SCI), although the specific clinical features that confer the greatest risks of renal failure are unknown. Now, long-term follow-up data are available from a cohort of 73 patients with SCI, who require lifelong management of their bladder function, with a median follow-up duration of 41 years.

In this cohort, 33 patients underwent $>1$ pressure-flow urodynamic evaluation in the period following SCI. All cystometric data obtained from such recordings were retrospectively analysed and examined for possible associations with renal deterioration, which occurred in $26 \%$ of patients in the cohort. No statistically significant associations were observed between a range of urodynamic parameters and renal deterioration, including the presence of detrusor-sphincter dyssynergia, detrusor or intravesical pressures during the filling and/or voiding phases, post-void residual volume, maximum bladder capacity, and bladder compliance. Detrusor overactivity (DO) was calculated as a ratio of the total duration of detrusor contractions during cystoscopy relative to the total duration of the cystometry recording (DO:cystometry ratio), and patients were classified into one of three groups on the basis of this ratio $(<0.33,0.33-0.66$ and $>0.66)$. A cumulative risk of deterioration in renal function (defined as a $\leq 30 \%$ decline in glomerular filtration rate (GFR) on investigation of split renal function or a $\leq 51 \%$ decline in relative GFR) of $40 \%$ was observed in patients with a DO:cystometry ratio $>0.33$, versus $12 \%$ of those with a ratio $<0.33$, indicating that the extent of detrusor overactivity is a significant predictor of renal deterioration.

These findings highlight the importance of management strategies intended to reduce the extent of DO in patients with a previous SCI.

Peter Sidaway

ORIGINAL ARTICLE Elmelund M. et al. Renal deterioration after spinal cord injury is associated with length of detrusor contractions during cystometry-A study with a median of 41 years follow-up. Neurourol. Urodyn. http://dx.doi.org/10.1002/ nau.23163 (2016) 